\title{
Physical activity, sedentary behaviour and colorectal cancer risk in the UK Biobank
}

\author{
Jessica S Morris ${ }^{1}$, Kathryn E Bradbury ${ }^{2}$, Amanda J Cross ${ }^{1}$, Marc J Gunter ${ }^{3}$ and Neil Murphy ${ }^{\star}, 3$ \\ ${ }^{1}$ Department of Epidemiology and Biostatistics, School of Public Health, Imperial College London, London, UK; ${ }^{2}$ Cancer \\ Epidemiology Unit, Nuffield Department of Population Health, University of Oxford, Oxford, UK and ${ }^{3}$ Section of Nutrition and \\ Metabolism, International Agency for Research on Cancer, Lyon, France
}

Background: Observational studies have shown that physical activity levels are inversely, and sedentary behaviours are positively, associated with colorectal cancer risk; however, whether these relationships are consistent across anatomical subsites is uncertain.

\begin{abstract}
Methods: We investigated the associations between colorectal cancer and physical activity (metabolic equivalents (METs)-hours per week), and indicators of sedentary behaviour (television watching time and time spent using computers) among 430584 men and women enroled in the UK Biobank. Multivariable hazard ratios (HRs) and $95 \%$ confidence intervals (Cl) were estimated using Cox proportional hazards models.
\end{abstract}

Results: After a median follow-up time of 5.6 years, 2391 incident colorectal cancer cases were recorded. High ( $\geqslant 60-M E T$-hours per week) vs low (<10-MET-hours per week) total physical activity was associated with a lower colon cancer risk ( $\mathrm{HR}=0.84,95 \% \mathrm{Cl}$ : 0.72-0.98; p-trend $=0.04$ ), with comparable relationships observed for proximal and distal colon tumours, but no association for rectal cancer. Higher levels of television watching time were associated with greater colon cancer risk (HR for $\geqslant 5 \mathrm{~h}$ per day vs $\leqslant 1 \mathrm{~h}$ per day $=1.32,95 \% \mathrm{Cl}$ : 1.04-1.68; p-trend=0.007). Time spent using computers was not associated with colorectal cancer risk.

Conclusions: Higher levels of physical activity were associated with lower colon cancer risk, with no heterogeneity by colonic subsite. Sedentary behaviour (television watching) was associated with elevated colon cancer risk.

Colorectal cancer is the third most commonly diagnosed cancer in men and the second in women worldwide (IARC-WHO, 2015). A large body of evidence has shown that greater levels of physical activity are associated with a lower risk of colorectal cancer (WCRF-AICR, 2011; Moore et al, 2016). However, colorectal cancers are a heterogeneous collection of tumours with variable molecular characteristics, and it is hypothesised that tumours located at different anatomical subsites may have distinct aetiological risk factors (Wei et al, 2004; Limsui et al, 2010). For physical activity, generally consistent inverse relationships have been found for colon and rectal cancers, with a recent participantlevel pooled analysis, which included 1.44 million participants, reporting that leisure-time physical activity was associated with lower risks of colon cancer and rectal cancer when analysed separately (Moore et al, 2016). It is unclear, however, how physical activity levels are related to colon cancer risk across the proximal and distal anatomical regions as few studies have been of sufficient size to undertake analyses by subsite, and inconsistent results have emerged. For instance, in the European Prospective Investigation into Cancer and Nutrition (EPIC), an inverse relationship was reported only between total physical activity and proximal colon cancer risk, but null results were found for distal colon cancer risk (Friedenreich et al, 2006). In contrast, in other large prospective studies, inverse relationships of similar magnitude have been observed across the colonic subsites (Howard et al, 2008; Burón Pust et al, 2017; Wei et al, 2017).

Sedentary behaviours, defined as sitting or reclined posture activities which expend $\leqslant 1.5$ metabolic equivalents (METs) (Owen et al, 2000), may also influence colorectal cancer risk. In the National Institutes of Health (NIH)-AARP Diet and Health

*Correspondence: Dr N Murphy; E-mail: murphyn@iarc.fr

Received 20 September 2017; revised 19 December 2017; accepted 20 December 2017; published online 8 March 2018

(C) 2018 Cancer Research UK. All rights reserved 0007 - 0920/18 
Study, sedentary behaviour (time spent watching television and videos) was associated with a greater colorectal cancer risk (Howard et al, 2008). A meta-analysis of case-control and cohort studies reported that total sitting time, television viewing time, and occupational sitting time were all positively associated with colon cancer development (Schmid and Leitzmann, 2014). However, whether this relationship differs within the colon for proximal and distal tumours has been largely unstudied.

In the current analysis, we investigated how total physical activity levels and sedentary behaviours, such as television watching and time spent using computers, are related to colorectal cancer risk in the UK Biobank study. The UK Biobank is a largescale prospective cohort study including over 500000 participants. The large number of recorded colorectal cancer cases provides substantial statistical power to investigate relationships across colorectal cancer subsites (colon, proximal colon, distal colon, and rectum) and according to sex and body habitus.

\section{MATERIALS AND METHODS}

Study participants. The UK Biobank is a prospective cohort study which aims to investigate the genetic, lifestyle, and environmental causes of a range of diseases (UK-Biobank, 2010; Allen et al, 2012). Between 2006 and 2010, 502656 adults aged between 40 and 69 years (229182 men and 273474 women) were recruited. All participants were registered with the UK National Health Service (NHS) and lived within $\sim 25$ miles $(40 \mathrm{~km})$ of 1 of the 22 study assessment centres. The UK Biobank invited $\sim 9.2$ million people to participate through postal invitation with a telephone follow-up, with a response rate of 5.7\%. The UK Biobank has approval from the North West Multi-centre Research Ethics Committee, the National Information Governance Board for Health and Social Care in England and Wales, and the Community Health Index Advisory Group in Scotland. In addition, an independent Ethics and Governance Council was formed in 2004 to oversee UK Biobank's continuous adherence to the Ethics and Governance Framework, which were developed for the study (http://www.ukbiobank.ac.uk/ethics/). All participants provided written informed consent.

During the baseline recruitment visit, participants were asked to complete a self-administered touchscreen questionnaire, which included questions on socio-demographics (including age, sex, education and Townsend deprivation score), health and medical history, lifestyle exposures (including smoking habits, dietary intakes, and alcohol consumption), early life exposures, and medication use. At the baseline interview, participants also underwent physical measurements, including body weight, height, and waist circumference. Exclusions prior to the onset of analyses included: participants with prevalent cancer at recruitment $(n=27058)$ and those with missing self-reported physical activity information and body size measurements $(n=45014)$. Our analysis therefore included 430584 participants (201225 men and 229359 women).

Assessment of exposure. The questions on physical activity that were included in the UK Biobank baseline questionnaire were adapted from the International Physical Activity Questionnaire (IPAQ) short form, a validated survey instrument (Craig et al, 2003). Questions are listed in Supplementary Table 1 and are available to view on the UK Biobank Web site (http://biobank.ctsu.ox.ac.uk/crystal/label.cgi?id=100054). The questions captured the frequency and duration of three different levels of activity (walking, moderate, and vigorous). Participants were asked on how many days in a typical week they engaged in each activity level for 10 min or more. For each level in which an answer of one or more days was given, the participant was then asked how many minutes they usually spent doing that activity on a typical day. Using this information, METs were used to quantify the intensity of physical activity. The MET values represent the ratio of energy expended per kilogram of body weight per hour to the standard resting metabolic rate (Ainsworth et al, 2011). The number of days per week that participants engaged in each level of physical activity was multiplied by the number of minutes spent per day doing that activity. This gave the total number of minutes spent per week engaged in each activity category. Total MET values for each category from the International Physical Activity Questionnaire short form were: 3.3 for walking, 4.0 for moderate physical activity and 8.0 for vigorous physical activity (IPAQ Research Committee, 2005). We report excess METs, which are calculated by subtracting one MET from the value for each activity, and represent the energy expenditure above that of an inactive person (Howley, 2001). Excess MET values were therefore 2.3 for walking, 3.0 for moderate physical activity and 7.0 for vigorous physical activity. Excess MET-hours per week were calculated by multiplying the excess MET value for each activity by the duration of activity in hours per week (IPAQ Research Committee, 2005). In the baseline questionnaire, participants were also asked in a typical day how many hours they spent watching television and using a computer. This information was used as an indicator of sedentary behaviours as television watching and time spent using computers are two of the most wide-spread leisure-time sedentary behaviours in adults (Office for National Statistics, 2006)

Assessment of outcome. Prevalent and incident cancer cases within the UK Biobank cohort were identified through linkage to national cancer registries. Complete follow-up was available through 30th November 2014 for England and Wales and 31st December 2014 for Scotland. Cancer incidence data were coded using the 10th Revision of the International Classification of Diseases (ICD-10). Proximal colon cancers included those found within the caecum, appendix, ascending colon, hepatic flexure, transverse colon, and splenic flexure (C18.0-18.5). Distal colon cancers included those found within the descending (C18.6) and sigmoid (C18.7) colon. Overlapping (C18.8) and unspecified (C18.9) lesions of the colon were included in colon cancers only. Cancer of the rectum included cancers occurring at the recto sigmoid junction (C19) and rectum (C20).

Statistical analysis. Hazard ratios (HRs) and 95\% confidence intervals (CIs) were estimated using Cox proportional hazards models. Age was the primary time variable in all models. Time at entry was age at recruitment. Exit time was age at whichever of the following came first: colorectal cancer diagnosis, death, or the last date at which follow-up was considered complete. Models were stratified by age at recruitment in 5-year categories, Townsend deprivation index fifths, and region of the recruitment assessment centre. Deviations from proportionality was assessed using an analysis of Schoenfeld residuals (Schoenfeld, 1982), with no evidence of non-proportionality being detected. Participants were grouped into predefined categories for total physical activity levels $(<10,10-<20,20-<40,40-<60, \geqslant 60$ MET hours per week), television watching time $(\leqslant 1,2-3,4-5,>5 \mathrm{~h}$ per day for sexes combined analyses, and $\leqslant 1,2-3, \geqslant 4 \mathrm{~h}$ per day for sex-specific analyses), and time spent using computers (none, $>0-1,2-3$, $\geqslant 4 \mathrm{~h}$ per day for sexes combined analyses, and none, $>0-1, \geqslant 2 \mathrm{~h}$ per day for sex-specific analyses).

The multivariable models were adjusted for a set of a prioridetermined colorectal cancer risk factors, namely height (continuous, $\mathrm{cm}$ ), smoking status and intensity (never; former; current $-<15$ cigarettes per day; current $-\geqslant 15$ cigarettes per day; current - intensity unknown; unknown), alcohol consumption frequency (never; special occasions only; 1-3 times per month; 1-2 times per week; 3-4 times per week; daily or almost daily; unknown), family history of colorectal cancer (no; yes; unknown), prevalent diabetes (no; yes; unknown), regular aspirin/ibuprofen 
Table 1. Characteristics of study participants by category of total physical activity (MET-hours per week)

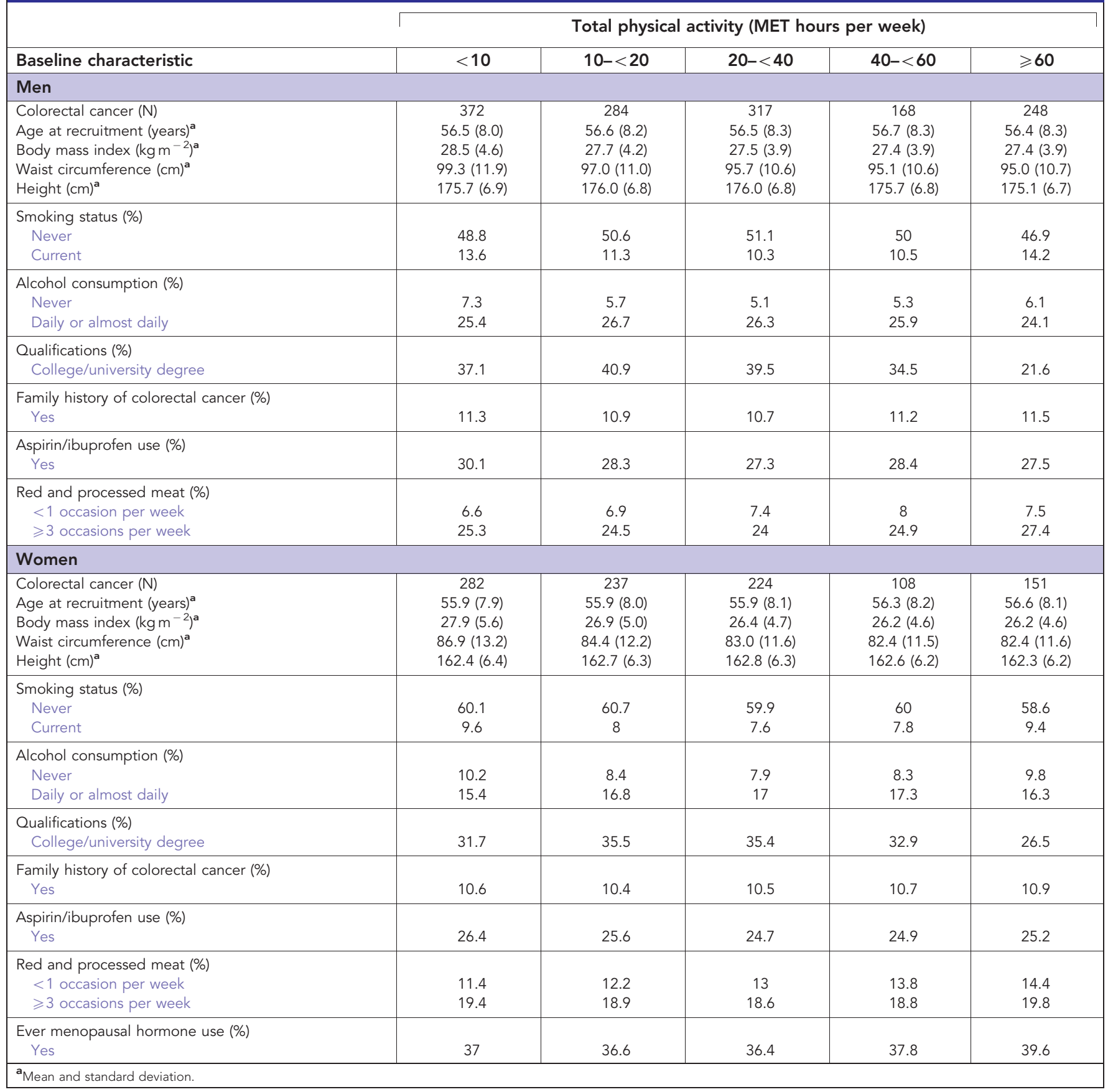

use (no; yes; unknown), qualifications (none; national exams at age 16 years (CSEs/O-levels/GCSEs or equivalent); vocational qualifications (NVQ/HND/HNC) or optional national exams at ages 1718 years (A-levels/AS-levels or equivalent); other professional qualifications; college/university degree; unknown, frequency of red and processed meat consumption ( $<2$ times per week; $2-3$ times per week; 3-4 times per week; $>4$ times per week; unknown), and, among women, ever use of hormone replacement therapy (HRT; no, yes, unknown). Further adjustment for previous colorectal cancer screening resulted in virtually unchanged risk estimates, so this variable was not included in the final multivariable models. As body size/adiposity is potentially on the causal pathway linking physical activity and sedentary activities with colorectal cancer, we also ran all models with and without adjustment for waist circumference. In addition, in sensitivity analyses, the television watching time and time spent using computers analyses were mutually adjusted for one another.

Trend tests across physical activity categories were calculated by assigning the median value of each measurement and modelling as continuous terms in Cox regression models. For television watching time and time spent using computers, trend tests were calculated by entering the categorical exposure variables into the models as continuous variables. As well as for overall colorectal cancer, analyses were undertaken for colon cancer, proximal colon cancer, distal colon cancer, and rectal cancer for sexes combined and for men and women separately. Heterogeneity of associations by sex and across anatomical cancer subsites was assessed by calculating $X^{2}$ statistics.

The physical activity and colorectal cancer associations were further assessed across subgroups of body mass index (BMI; $<25$, 
Table 2. Risk (hazard ratios) of colorectal cancer associated with total physical activity

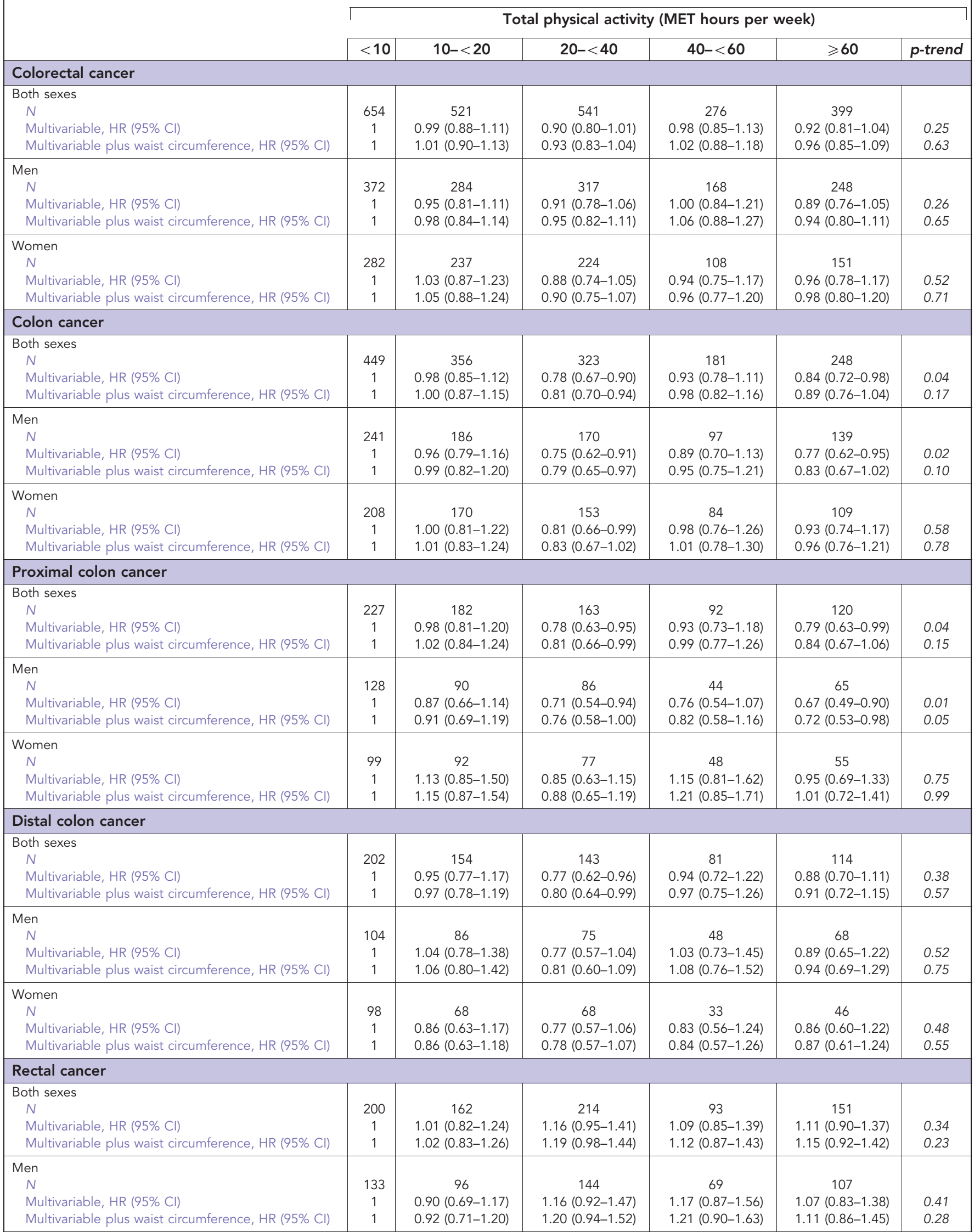




\begin{tabular}{|c|c|c|c|c|c|c|}
\hline & \multicolumn{6}{|c|}{ Total physical activity (MET hours per week) } \\
\hline & $<10$ & $10-<20$ & $20-<40$ & $40-<60$ & $\geqslant 60$ & p-trend \\
\hline $\begin{array}{l}\text { Women } \\
\text { N } \\
\text { Multivariable, HR }(95 \% \mathrm{CI}) \\
\text { Multivariable plus waist circumference, HR }(95 \% \mathrm{CI})\end{array}$ & $\begin{array}{c}67 \\
1 \\
1\end{array}$ & $\begin{array}{c}66 \\
1.21(0.86-1.71) \\
1.22(0.87-1.72)\end{array}$ & $\begin{array}{c}70 \\
1.15(0.82-1.61) \\
1.16(0.83-1.63)\end{array}$ & $\begin{array}{c}24 \\
0.89(0.56-1.42) \\
0.90(0.56-1.44)\end{array}$ & $\begin{array}{c}44 \\
1.18(0.80-1.72) \\
1.19(0.81-1.75)\end{array}$ & $\begin{array}{l}0.78 \\
0.74\end{array}$ \\
\hline $\begin{array}{l}\text { Abbreviations: } \mathrm{Cl}=\text { confidence interval; } \mathrm{HR}=\text { hazard ratio. Multivari } \\
\text { cigarettes per day; current - intensity unknown; unknown), alcoho } \\
\text { daily; unknown), family history of colorectal cancer (no; yes; unkn } \\
\text { levels/GCSEs or equivalent; NVQ/HND/HNC/A-levels/AS-levels o } \\
\text { (no, yes, unknown), frequency of red and processed meat consun } \\
\text { year categories), Townsend deprivation index fifths, and region }\end{array}$ & $\begin{array}{l}\text { nsumpti } \\
\text { ), preva } \\
\text { uivalen } \\
\text { on }(<2\end{array}$ & $\begin{array}{l}\text { xx regression using } \\
\text { frequency (never; sp } \\
\text { t diabetes (no; yes; } \\
\text { ther professional qu } \\
\text { es per week; } 2-3 \text { tin }\end{array}$ & $\begin{array}{l}\text { ng status and intensi } \\
\text { occasions only; } 1-3 \mathrm{t} \\
\text { own), regular aspirin/ } \\
\text { tions; college/univer } \\
\text { r week; } 3-4 \text { times/w }\end{array}$ & $\begin{array}{l}\text { ever; former; current } \\
\text { /month; } 1-2 \text { times/w } \\
\text { profen use (no; yes; } \\
\text { degree; unknown), ev } \\
\geqslant 4 \text { times per week; }\end{array}$ & $\begin{array}{l}\text { cigarettes per day; } \\
\text { wn), qualifications ( } \\
\text { of hormone replace } \\
\text { own), and stratified b }\end{array}$ & $\begin{array}{l}\text { urrent - } \geqslant 15 \\
\text { ily or almost } \\
\text { ne; CSEs/O- } \\
\text { nent therapy } \\
\text { sex, age (5- }\end{array}$ \\
\hline
\end{tabular}

$25-<30, \geqslant 30 \mathrm{~kg} \mathrm{~m}^{-2}$ ), waist circumference (sex-specific thirds), smoking status (never, former, current), and television watching time $(\leqslant 1,2-3, \geqslant 4 \mathrm{~h}$ per day). Interaction terms (multiplicative scale) between these variables and physical activity were included in separate models. The statistical significance of the cross-product terms were evaluated using the likelihood ratio test. Similarly, the television watching time and colorectal cancer relationship was further assessed across subgroups of time spent using computers each day (none, anytime). To assess the possibility of reverse causality, cases diagnosed within the first two years of follow-up were excluded from all of the analyses. We repeated the analyses with multiple imputations to account for missing total physical activity information; however no differences were detected with the complete case analysis results presented (data not shown).

Statistical tests were all two-sided and a $P$-value of $<0.05$ was considered statistically significant. Analyses were conducted using Stata version 13.1.

\section{RESULTS}

After a median follow-up time of 5.6 years, 2391 cases of colorectal cancer (1389 in men and 1002 in women) were recorded. Of these cases, 1557 were colon tumours (784 proximal colon, 694 distal colon, and 79 overlapping or unspecified), 820 were rectal tumours, and 14 were overlapping between the colon and rectum. Compared with those in the lowest category $(<10$ MET hours per week), men and women in the highest total physical activity category ( $\geqslant 60$ MET hours per week) had lower BMI and waist circumferences, were less likely to have attained a college education or university degree, to be never smokers, and to be regular aspirin/ ibuprofen users (Table 1).

\section{Physical activity}

Colorectal cancer. There was no association between physical activity and colorectal cancer risk in the sexes-combined multivariable model (HR for $\geqslant 60 v s<10$ MET hours per week $=0.92$, 95\% CI: $0.81-1.04 ; p$-trend $=0.25)$, with similar relationships found for men and women $(p$-interaction $=0.57)($ Table 2$)$.

Colon cancer. For colon cancer, in the sexes-combined model, the highest physical activity (METs) category had a $16 \%$ lower risk compared to those in the lowest category (HR for $\geqslant 60 v s<10$ MET hours per week $=0.84,95 \% \mathrm{CI}$ : 0.72-0.98; $p$-trend $=0.04)$. A statistically significant inverse association between physical activity and colon cancer risk was found only among men (HR for $\geqslant 60 v \mathrm{~s}$ $<10$ MET hours per week $=0.77,95 \%$ CI: $0.62-0.95 ; p$ trend $=0.02$ ), but not women (HR for $\geqslant 60$ vs $<10$ MET hours per week $=0.93$, 95\% CI: 0.74-1.17; $p$-trend $=0.58)$; however, this difference between sexes did not reach statistical significance $(p$ interaction $=0.25$ ).

For the sexes-combined models, no heterogeneity by colonic subsite was found $(p$-heterogeneity $=0.52)$, with a statistically significant inverse association observed between total physical activity and proximal colon cancer risk (HR for $\geqslant 60 v s<10$ MET hours per week $=0.79,95 \% \mathrm{CI}$ : $0.63-0.99 ; p$-trend $=0.04)$, and a non-significant inverse association for distal colon cancer risk (HR for $\geqslant 60$ vs $<10$ MET hours per week $=0.88$, 95\% CI: $0.70-1.11$; $p$-trend $=0.38)$. Among men, a statistically significant inverse association was found between total physical activity and proximal colon cancer risk (HR for $\geqslant 60$ vs $\leqslant 10$ MET hours per week $=0.67,95 \%$ CI: $0.49-0.90$; $p$-trend $=0.01)$, but no relationship was observed for distal colon cancer risk. Among women, no significant associations were observed between total physical activity and risks proximal colon and distal colon. The differences between the sexes did not reach statistical significance for proximal colon cancer $(p$-interaction $=0.15)$ and distal colon cancer $(p$ interaction $=0.89$ ).

Additional statistical adjustment for waist circumference slightly attenuated the physical activity associations for colon cancer and its subsites (attenuated the HR for the $\geqslant 60$ MET hours per week group by between 2 and 8\%), and rendered the HRs in this physical activity group no longer statistically significant for all models, except for proximal colon cancer among men (HR for $\geqslant 60 v s<10$ MET hours per week $=0.72$, 95\% CI: 0.53-0.98) (Table 2).

Rectal cancer. There was no relationship observed between physical activity and risk of rectal cancer for sexes-combined models, and for when men and women were analysed separately $(p$-interaction $=0.69)$.

Television watching time and time spent using computers and colorectal cancer risk

Colorectal cancer. In the sexes-combined multivariable model, higher levels of television watching time was associated with a greater colorectal cancer risk (HR for $>5 \mathrm{~h}$ per day $v s \leqslant 1 \mathrm{~h}$ per day $=1.26,95 \%$ CI: $1.04-1.53$; $p$-trend $=0.01) \quad($ Table 3$)$. A statistically significant positive association between television watching time and colorectal cancer was only found for men $(\mathrm{HR}$ for $\geqslant 4 \mathrm{~h}$ per day $v s \leqslant 1 \mathrm{~h}$ per day $=1.35$, 95\% CI: $1.13-1.61$; $p$-trend $=0.02$ ), but not women (HR for $\geqslant 4 \mathrm{~h} /$ day $v s \leqslant 1 \mathrm{~h} /$ day $=1.11,95 \%$ CI: $0.91-1.35$; p-trend $=0.25)$; however, this difference between sexes did not reach statistical significance $(p$-interaction $=0.21)$. Additional statistical adjustment for waist circumference slightly attenuated the association between television watching time colorectal cancer $(5.5 \%$ reduction in HR of the $\geqslant 5 \mathrm{~h}$ per day group) and rendered the HRs in this television watching time group to be no longer statistically significant. Time 
Table 3. Risk (hazard ratios) of colorectal cancer associated with television watching time

Television watching time

\section{Colorectal cancer}

\begin{tabular}{|c|c|c|c|c|c|}
\hline $\begin{array}{l}\text { Both sexes } \\
N \\
\text { Multivariable, HR }(95 \% \mathrm{Cl}) \\
\text { Multivariable plus waist circumference, HR }(95 \% \mathrm{Cl})\end{array}$ & $\begin{array}{c}\leqslant 1 \text { h per day } \\
373 \\
1 \\
1\end{array}$ & $\begin{array}{c}2-3 \text { h per day } \\
1186 \\
1.17(1.04-1.32) \\
1.15(1.02-1.29)\end{array}$ & $\begin{array}{l}4-5 \text { h per day } \\
663 \\
1.24(1.08-1.41) \\
1.19(1.04-1.36)\end{array}$ & $\begin{array}{c}>5 \text { h per day } \\
159 \\
1.26(1.04-1.53) \\
1.19(0.98-1.45)\end{array}$ & $\begin{array}{l}\text { p-trend } \\
0.01 \\
0.07\end{array}$ \\
\hline $\begin{array}{l}\text { Men } \\
N \\
\text { Multivariable, HR }(95 \% \mathrm{Cl}) \\
\text { Multivariable plus waist circumference, HR }(95 \% \mathrm{Cl})\end{array}$ & $\begin{array}{c}\leqslant 1 \text { h per day } \\
196 \\
1 \\
1\end{array}$ & $\begin{array}{l}2-3 \text { h per day } \\
685 \\
1.24(1.05-1.46) \\
1.21(1.03-1.42)\end{array}$ & $\begin{array}{l}\geqslant 4 \text { h per day } \\
501 \\
1.35(1.13-1.61) \\
1.28(1.07-1.53)\end{array}$ & & $\begin{array}{r}\text { p-trend } \\
0.02 \\
0.09\end{array}$ \\
\hline $\begin{array}{l}\text { Women } \\
\mathrm{N} \\
\text { Multivariable, HR }(95 \% \mathrm{Cl}) \\
\text { Multivariable plus waist circumference, HR }(95 \% \mathrm{Cl})\end{array}$ & $\begin{array}{c}\leqslant 1 \text { h per day } \\
177 \\
1 \\
1\end{array}$ & $\begin{array}{l}\text { 2-3 h per day } \\
501 \\
1.09(0.92-1.30) \\
1.08(0.90-1.29)\end{array}$ & $\begin{array}{l}\geqslant 4 \text { h per day } \\
321 \\
1.11(0.91-1.35) \\
1.08(0.88-1.32)\end{array}$ & & $\begin{array}{r}\text { p-trend } \\
0.25 \\
0.40\end{array}$ \\
\hline
\end{tabular}

\section{Colon cancer}

\section{Both sexes}

N

Multivariable, HR $(95 \% \mathrm{Cl})$

Multivariable plus waist circumference, HR (95\% Cl)

Men

N

Multivariable, HR (95\% CI)

Multivariable plus waist circumference, HR (95\% Cl) Women

N

Multivariable, HR (95\% Cl)

Multivariable plus waist circumference, $\operatorname{HR}(95 \% \mathrm{Cl})$

\begin{tabular}{|c|c|}
$\leqslant 1$ h per day & $2-3 \mathrm{~h}$ per day \\
246 & 753 \\
1 & $1.14(0.98-1.32)$ \\
1 & $1.11(0.96-1.29)$ \\
$\leqslant 1 \mathrm{~h} / \mathrm{d}$ & $2-3 \mathrm{~h}$ per day \\
111 & 408 \\
1 & $1.31(1.05-1.62)$ \\
1 & $1.27(1.02-1.57)$ \\
$\leqslant 1 \mathrm{~h} / \mathrm{d}$ & $2-3 \mathrm{~h}$ per day \\
135 & 345 \\
1 & $1.00(0.82-1.23)$ \\
1 & $0.98(0.80-1.21)$
\end{tabular}

4-5 h per day 441

$1.25(1.06-1.47)$

$1.19(1.01-1.41)$

$\geqslant 4 \mathrm{~h}$ per day 311

$1.45(1.15-1.83)$

1.37 (1.08-1.72)

$\geqslant 4 \mathrm{~h}$ per day 241

$1.09(0.87-1.37)$

$1.05(0.84-1.33)$

\begin{tabular}{|c|c}
$>5$ h per day & p-trend \\
111 & \\
$1.32(1.04-1.68)$ & 0.007 \\
$1.23(0.97-1.57)$ & 0.045 \\
& p-trend \\
& 0.02 \\
& 0.09 \\
& p-trend \\
& 0.16 \\
& 0.28
\end{tabular}

\section{Proximal colon cancer}

\section{Both sexes}

N

Multivariable, HR (95\% Cl)

Multivariable plus waist circumference, HR (95\% CI) Men

N

Multivariable, HR (95\% Cl)

Multivariable plus waist circumference, HR (95\% Cl) Women

N

Multivariable, HR $(95 \% \mathrm{Cl})$

Multivariable plus waist circumference, HR (95\% Cl)

\section{Distal colon cancer}

\section{Both sexes}

N

Multivariable, HR (95\% Cl)

Multivariable plus waist circumference, HR (95\% Cl)

Men

N

Multivariable, HR $(95 \% \mathrm{Cl})$

Multivariable plus waist circumference, HR (95\% Cl)

Women

N

Multivariable, HR $(95 \% \mathrm{CI})$

Multivariable plus waist circumference, HR (95\% Cl)

\begin{tabular}{|c|c|c|}
\hline$\leqslant 1 \mathrm{~h}$ per day & $2-3 \mathrm{~h}$ per day & $4-5 \mathrm{~h}$ per day \\
127 & 356 & 239 \\
1 & $1.04(0.84-1.27)$ & $1.28(1.02-1.61)$ \\
1 & $1.00(0.81-1.23)$ & $1.20(0.96-1.52)$ \\
$\leqslant 1 \mathrm{~h}$ per day & $2-3 \mathrm{~h}$ per day & $\geqslant 4 \mathrm{~h}$ per day \\
58 & 191 & 162 \\
1 & $1.18(0.88-1.60)$ & $1.45(1.05-1.99)$ \\
1 & $1.14(0.84-1.54)$ & $1.35(0.98-1.86)$ \\
$\leqslant 1 \mathrm{~h}$ per day & $2-3 \mathrm{~h}$ per day & $\geqslant 4 \mathrm{~h}$ per day \\
69 & 165 & 135 \\
1 & $0.91(0.68-1.22)$ & $1.14(0.83-1.57)$ \\
1 & $0.88(0.66-1.18)$ & $1.08(0.78-1.48)$
\end{tabular}

\begin{tabular}{|c|c}
$>5$ h per day & p-trend \\
58 & \\
$1.29(0.93-1.80)$ & 0.009 \\
$1.18(0.85-1.65)$ & 0.047 \\
& p-trend \\
& 0.03 \\
& 0.08 \\
& p-trend \\
& 0.16 \\
& 0.30
\end{tabular}

\section{Rectal cancer}

N

Multivariable, HR $(95 \% \mathrm{Cl})$

Multivariable plus waist circumference, HR (95\% Cl)

Men

N

Multivariable, HR (95\% Cl)

Multivariable plus waist circumference, HR (95\% Cl)

Women

N

Multivariable, HR (95\% Cl)

Multivariable plus waist circumference, HR (95\% Cl)

\begin{tabular}{|c|c|c|}
$\leqslant 1 \mathrm{~h}$ per day & $2-3 \mathrm{~h}$ per day & $4-5 \mathrm{~h}$ per day \\
103 & 357 & 186 \\
1 & $1.29(1.03-1.62)$ & $1.26(0.97-1.62)$ \\
1 & $1.27(1.02-1.59)$ & $1.22(0.94-1.58)$ \\
$\leqslant 1 \mathrm{~h}$ per day & $2-3 \mathrm{~h}$ per day & $\geqslant 4 \mathrm{~h}$ per day \\
48 & 196 & 137 \\
1 & $1.43(1.04-1.98)$ & $1.45(1.02-2.06)$ \\
1 & $1.40(1.02-1.94)$ & $1.39(0.98-1.97)$ \\
$\leqslant 1 \mathrm{~h}$ per day & $2-3 \mathrm{~h}$ per day & $\geqslant 4 \mathrm{~h}$ per day \\
55 & 161 & 96 \\
1 & $1.16(0.85-1.59)$ & $1.09(0.76-1.56)$ \\
1 & $1.15(0.84-1.58)$ & $1.07(0.74-1.53)$
\end{tabular}

\begin{tabular}{|c|c}
\hline 5 h per day & p-trend \\
47 & \\
$1.33(0.92-1.92)$ & 0.28 \\
$1.27(0.88-1.83)$ & 0.44 \\
& p-trend \\
& 0.36 \\
& 0.54 \\
& \\
& 0.59 \\
& 0.66
\end{tabular}

\begin{tabular}{|c|c|c|}
\hline$\leqslant 1$ h per day & 2-3h per day & 4-5 h per day \\
\hline $\begin{array}{r}126 \\
1\end{array}$ & $\begin{array}{c}423 \\
1.22(0.99-1.50)\end{array}$ & $\frac{221}{24(099-156)}$ \\
\hline 1 & $1.21(0.99-1.48)$ & $1.21(0.96-1.53)$ \\
\hline $\begin{array}{c}1 \mathrm{~h} \text { per day } \\
84\end{array}$ & $\begin{array}{c}2-3 \text { h per day } \\
269\end{array}$ & $\begin{array}{c}\geqslant 4 \mathrm{~h} \text { per day } \\
192\end{array}$ \\
\hline 1 & $1.13(0.88-1.45)$ & $1.24(0.95-1.63)$ \\
\hline 1 & $1.11(0.87-1.43)$ & $1.20(0.91-1.58)$ \\
\hline$\leqslant 1$ h per day & $\begin{array}{c}2-3 \mathrm{~h} \text { per day } \\
154\end{array}$ & $\geqslant 4$ h per day \\
\hline 1 & 1.42 (0.99-2.01) & $1.15(0.77-1.73)$ \\
\hline 1 & 1.41 (0.99-1.99) & $1.14(0.76-1.71)$ \\
\hline
\end{tabular}

\begin{tabular}{|c|c}
$>5$ h per day & p-trend \\
46 & \\
$1.13(0.79-1.61)$ & 0.43 \\
$1.09(0.76-1.55)$ & 0.60 \\
& \\
& 0.29 \\
& 0.43 \\
& \\
& 0.87 \\
& 0.82
\end{tabular}

Abbreviations: $\mathrm{Cl}=$ confidence interval; $\mathrm{HR}=$ hazard ratio. Multivariable model: Cox regression using physical activity (MET hours per week; fifths), smoking status and intensity (never; former; current $-<15$ cigarettes/day; current - $\geqslant 15$ cigarettes per day; current - intensity unknown; unknown), alcohol consumption frequency (never; special occasions only; $1-3$ times/month; $1-2$ times per week; 3-4 times per week; daily or almost daily; unknown), family history of colorectal cancer (no; yes; unknown), prevalent diabetes (no; yes; unknown), regular aspirin/ibuprofen use (no; yes; unknown), qualifications (none; CSEs/O-levels/GCSEs or equivalent; NVQ/HND/HNC/A-levels/AS-levels or equivalent; other professional qualifications; college/university degree; unknown), ever use of hormone replacement therapy (no, yes, unknown), frequency of red and processed meat consumption ( $<2$ times per week; $2-3$ times/week; $3-4$ times/week; $\geqslant 4$ times per week; unknown), and stratified by sex, age (5-year categories), Townsend deprivation index fifths, and region of the recruitment assessment centre. 
Table 4. Risk (hazard ratios) of colorectal cancer associated with time spent on computers

\section{Colorectal cancer}

Both sexes

Multivariable, HR (95\% Cl)

Multivariable plus waist circumference, HR (95\% CI)

Men

N

Multivariable, HR $(95 \% \mathrm{Cl})$

Multivariable plus waist circumference, HR (95\% Cl)

Women

N

Multivariable, HR (95\% Cl)

Multivariable plus waist circumference, HR (95\% Cl)

\section{Time spent on computers}

\section{Colon cancer}

Both sexes

Multivariable, HR (95\% Cl)

Multivariable plus waist circumference, $\mathrm{HR}(95 \% \mathrm{Cl})$ Men

N

Multivariable, HR (95\% Cl)

Multivariable plus waist circumference, HR (95\% CI)

Women

N

Multivariable, HR (95\% Cl)

Multivariable plus waist circumference, HR (95\% Cl)

\begin{tabular}{|c|c} 
None & $0.1-1 \mathrm{~h}$ per day \\
1837 & 408 \\
1 & $0.95(0.85-1.06)$ \\
1 & $0.93(0.83-1.04)$ \\
None & $0.1-1 \mathrm{~h}$ per day \\
995 & 292 \\
1 & $0.98(0.86-1.12)$ \\
1 & $0.96(0.84-1.10)$ \\
None & $0.1-1 \mathrm{~h}$ per day \\
842 & 116 \\
1 & $0.90(0.74-1.10)$ \\
1 & $0.89(0.73-1.09)$
\end{tabular}

\begin{tabular}{|c}
$2-3 \mathrm{~h} / \mathrm{d}$ \\
92 \\
$1.07(0.87-1.32)$ \\
$1.04(0.84-1.29)$ \\
$\geqslant 2$ h per day \\
96 \\
$1.04(0.84-1.29)$ \\
$1.01(0.82-1.25)$ \\
$\geqslant 2$ h per day \\
36 \\
$1.03(0.73-1.44)$ \\
$1.01(0.72-1.41)$
\end{tabular}

\begin{tabular}{|c|c}
$\geqslant 4$ h per day & p-trend \\
40 & \\
$0.94(0.68-1.29)$ & 0.75 \\
$0.91(0.67-1.25)$ & 0.50 \\
& $p$-trend \\
& 0.89 \\
& 0.82 \\
& p-trend \\
& 0.58 \\
& 0.49
\end{tabular}

Proximal colon cancer

Both sexes

Multivariable, HR (95\% Cl)

Multivariable plus waist circumference, HR (95\% CI) Men

N

Multivariable, HR (95\% Cl)

Multivariable plus waist circumference, HR (95\% Cl) Women

N

Multivariable, HR (95\% CI)

Multivariable plus waist circumference, HR (95\% Cl)

\begin{tabular}{|c|c} 
None & $0.1-1 \mathrm{~h}$ per day \\
1199 & 263 \\
1 & $0.96(0.84-1.10)$ \\
1 & $0.94(0.82-1.08)$ \\
None & $0.1-1 \mathrm{~h}$ per day \\
599 & 171 \\
1 & $0.94(0.79-1.12)$ \\
1 & $0.92(0.77-1.09)$ \\
None & $0.1-1 \mathrm{~h}$ per day \\
600 & 92 \\
1 & $1.01(0.81-1.27)$ \\
1 & $1.00(0.80-1.25)$
\end{tabular}

2-3h per day

$1.12(0.86-1.45)$

$1.08(0.84-1.41)$

$\geqslant 2 \mathrm{~h}$ per day

59

$1.05(0.80-1.37)$

$1.00(0.76-1.32)$

$\geqslant 2 \mathrm{~h}$ per day

$1.01(0.68-1.52)$

$0.99(0.66-1.49)$

\begin{tabular}{|c|c}
$\geqslant 4 \mathrm{~h}$ per day & p-trend \\
23 & \\
$0.86(0.56-1.30)$ & 0.81 \\
$0.83(0.55-1.26)$ & 0.55 \\
& $p$-trend \\
& 0.83 \\
& 0.58 \\
& $p$-trend \\
& 0.98 \\
& 0.86
\end{tabular}

\section{Distal colon cancer}

\section{Both sexes}

Multivariable, HR $(95 \% \mathrm{Cl})$

Multivariable plus waist circumference, HR $(95 \% \mathrm{Cl})$ Men

Multivariable, HR (95\% Cl)

Multivariable plus waist circumference, HR (95\% CI)

Women

N

Multivariable, HR (95\% CI)

Multivariable plus waist circumference, HR (95\% Cl)

\begin{tabular}{|c|c} 
None & $0.1-1$ h per day \\
601 & 140 \\
1 & $1.04(0.86-1.25)$ \\
1 & $1.01(0.84-1.22)$ \\
None & $0.1-1$ h per day \\
292 & 92 \\
1 & $1.03(0.81-1.31)$ \\
1 & $1.00(0.79-1.27)$ \\
None & $0.1-1$ h per day \\
309 & 48 \\
1 & $1.06(0.78-1.44)$ \\
1 & $1.03(0.75-1.40)$
\end{tabular}

2-3 h per day

26

$0.97(0.65-1.44)$

$0.93(0.63-1.38)$

$\geqslant 2 \mathrm{~h}$ per day 27

$0.95(0.64-1.42)$

$0.91(0.61-1.35)$

$\geqslant 2 \mathrm{~h}$ per day 10

$0.84(0.45-1.59)$

$0.81(0.43-1.54)$

\begin{tabular}{|c|c}
\hline $\begin{array}{c}\text { i h per day } \\
11\end{array}$ & p-trend \\
$0.84(0.46-1.53)$ & 0.79 \\
$0.81(0.44-1.47)$ & 0.55 \\
& p-trend \\
& 0.86 \\
& 0.63 \\
& p-trend \\
& 0.77 \\
& 0.64
\end{tabular}

\section{Rectal cancer}

\section{Both sexes}

N

Multivariable, HR (95\% Cl)

Multivariable plus waist circumference, HR (95\% Cl) Men

Multivariable, HR (95\% Cl)

Multivariable plus waist circumference, HR (95\% Cl) Women

N

Multivariable, HR (95\% Cl)

Multivariable plus waist circumference, HR $(95 \% \mathrm{Cl})$ $\operatorname{HR}(95 \% \mathrm{Cl})$

\begin{tabular}{|c|c|c} 
None & $0.1-1 \mathrm{~h}$ per day & $2-3 \mathrm{~h}$ per day \\
532 & 114 & 32 \\
1 & $0.93(0.75-1.14)$ & $1.31(0.91-1.88)$ \\
1 & $0.91(0.74-1.12)$ & $1.28(0.89-1.84)$ \\
None & $0.1-1 \mathrm{~h}$ per day & $\geqslant 2 \mathrm{~h}$ per day \\
278 & 72 & 30 \\
1 & $0.85(0.66-1.11)$ & $1.18(0.81-1.74)$ \\
1 & $0.84(0.65-1.10)$ & $1.15(0.78-1.69)$ \\
None & $0.1-1 \mathrm{~h}$ per day & $\geqslant 2 \mathrm{~h}$ per day \\
254 & 42 & 14 \\
1 & $1.09(0.78-1.52)$ & $1.31(0.76-2.26)$ \\
1 & $1.08(0.78-1.51)$ & $1.30(0.75-2.24)$
\end{tabular}

\begin{tabular}{|c|c|}
\hline$\geqslant 4$ h per day & p-trend \\
12 & \\
$1.00(0.56-1.79)$ & 0.62 \\
$0.98(0.55-1.75)$ & 0.74 \\
& p-trend \\
& 0.91 \\
& 0.96 \\
& p-trend \\
& 0.36 \\
& 0.39
\end{tabular}

Abbreviations: $\mathrm{Cl}=$ confidence interval; $\mathrm{HR}=$ hazard ratio. Multivariable model: Cox regression using physical activity (MET hours per week; fifths), smoking status and intensity (never; former; current - $<15$ cigarettes per day; current - $\geqslant 15$ cigarettes per day; current - intensity unknown; unknown), alcohol consumption frequency (never; special occasions only; $1-3$ times per month; 1-2 times per week; 3-4 times per week; daily or almost daily; unknown), family history of colorectal cancer (no; yes; unknown), prevalent diabetes (no; yes; unknown), regular aspirin/ibuprofen use (no; yes; unknown), qualifications (none; CSEs/O-levels/GCSEs or equivalent; NVQ/HND/HNC/A-levels/AS-levels or equivalent; other professional qualifications; college/university degree; unknown), ever use of hormone replacement therapy (no, yes, unknown), frequency of red and processed meat consumption ( $<2$ times per week; $2-3$ times/week; $3-4$ times/week; $\geqslant 4$ times/ week; unknown), and stratified by sex, age (5-year categories), Townsend deprivation index fifths, and region of the recruitment assessment centre. 
spent using computers was not associated with risk of colorectal cancer (Table 4), and this relationship did not differ after additional adjustment for television watching time (Supplementary Table 3).

Colon cancer. In the sexes-combined multivariable model, higher levels of television watching time was associated with a greater colon cancer risk (HR for $>5 \mathrm{~h}$ per day $v s \leqslant 1 \mathrm{~h}$ per day $=1.32$, 95\% CI: $1.04-1.68 ; p$-trend $=0.007$ ) (Table 3). This positive relationship was only present among men, and not women, although this difference did not reach statistical significance $(p$ interaction $=0.10)$. The sexes-combined association between television watching time and colon cancer risk did not differ by subsite with HRs of similar strength observed for proximal and distal colon cancers $(p$-heterogeneity $=0.93) \quad($ Table 3$)$. The positive relationships between television watching time and proximal and distal colon cancer were stronger and statistically significant for men than for women; however, these differences did not reach statistical significance (proximal colon $p$ interaction $=0.31$; distal colon $p$-interaction $=0.28$ ). Similar relationships between television watching time and colon cancer were observed after additional statistical adjustment for time spent on computers (Supplementary Table 2). Time spent using computers was not associated with risk of colon cancer, proximal colon cancer, and distal colon cancer (Table 4), and these relationships did not differ after additional adjustment for television watching time (Supplementary Table 3). Additional statistical adjustment for waist circumference slightly attenuated the association between television watching time and cancers of the colon and proximal colon (the HRs were attenuated for the $\geqslant 5 \mathrm{~h}$ per day group by between 6 and 8\%), and rendered the HRs in this television watching time group to be no longer statistically significant.

Rectal cancer. There were no relationships observed between television watching time and time spent using computers and rectal cancer risk in the sexes-combined models, and for when men and women were analysed separately (television watching time $p$ interaction $=0.77$; time spent using computers $p$-interaction $=0.70$ ).

Sensitivity and subgroup analyses. The associations between total physical activity and colorectal cancer were similar across subgroups of BMI $(p$-interaction $=0.61)$, waist circumference $(p$ interaction $=0.93)$, smoking status $(p$-interaction $=0.14)$, and television watching time $(p$-interaction $=0.24)$ (data not shown). The relationship between television watching time and colorectal cancer did not differ across subgroups of time spent using computers $(p$-interaction $=0.23)$. Similar relationships were also observed when colorectal cancer cases which occurred during the first two years of follow-up were excluded from the analyses (data not shown).

\section{DISCUSSION}

In this prospective analysis of UK Biobank participants, total physical activity levels were inversely associated with colon cancer risk. This relationship was most apparent among men and for proximal colon cancer. Conversely, sedentary behaviour, as indicated by television watching time, was associated with elevated colon cancer risk. Total physical activity and the sedentary behaviours were not associated with rectal cancer risk in this population.

Our finding that total physical activity was associated with lower colon cancer risk is in accordance with a large body of epidemiological evidence (Friedenreich et al, 2006; Wolin et al, 2009; Moore et al, 2016). This relationship was consistent across body habitus and smoking status subgroups, and was most apparent for men compared to women, although this difference was not statistically significant. Consistent with other individual prospective analyses (Lee et al, 2007; Burón Pust et al, 2017; Wei et al, 2017), we found no relationship between total physical activity and rectal cancer risk. However, a recent participant-level pooled analysis which included 5531 rectal cancer cases reported an inverse relationship between physical activity and rectal cancer incidence (highest $v s$ lowest leisure-time physical activity group, relative risk $(\mathrm{RR})=0.87,95 \% \mathrm{CI}: 0.80-0.95)$ (Moore et al, 2016), which suggests that the null results reported by individual studies such as the current analysis ( $n=820$ rectal cancer cases) may be a consequence of insufficient sample size to detect weak-to-moderate associations.

For colon cancer, although we observed statistically significant inverse relationships for tumours in the proximal region and not the distal region with physical activity, this heterogeneity did not differ statistically. These results are generally consistent with those reported by other large prospective studies which did not observe heterogeneous physical activity relationships across the proximal and distal colon regions (Howard et al, 2008; Burón Pust et al, 2017; Wei et al, 2017), with the notable exception of the EPIC study (Friedenreich et al, 2006). Recently, in the Million's Women Study, which included 12761 colon cancer cases, similar magnitude inverse relationships were observed between strenuous exercise and proximal and distal colon cancers (Burón Pust et al, 2017). Similarly, in the Nurses' Health Study and the NIH-AARP Diet and Health Study, lower risks were generally observed between various indicators of physical activity and tumours in both colonic regions (Howard et al, 2008; Wei et al, 2017).

The results of our study contribute to a growing body of evidence which has reported sedentary behaviour as a risk factor for the development of colon cancer, independent of physical activity levels (Howard et al, 2008; Schmid and Leitzmann, 2014). A recent meta-analysis reported that when comparing the highest with the lowest total sitting time group, a $24 \%$ greater risk $(\mathrm{RR}=1.24,95 \% \mathrm{CI}: 1.03-1.50)$ of developing colon cancer was observed (Schmid and Leitzmann, 2014). Consistent with the findings from our analysis, for television watching time the same meta-analysis reported a $54 \%$ elevated risk $(\mathrm{RR}=1.54,95 \% \mathrm{CI}$ : 1.19-1.98) of colon cancer when the highest and lowest viewing time groups were compared (Schmid and Leitzmann, 2014). Television viewing time has been shown to be accompanied with other lifestyle behaviours, such as smoking and eating energy dense foods (Wiecha et al, 2006; Schmid and Leitzmann, 2014), which are also related to cancer risk (Schmid and Leitzmann, 2014). We did not, however, observe any relationship between time spent on computers and colorectal cancer risk. It has been previously shown that for sedentary behaviour questionnaires, television watching assessment has higher test-retest reliability than computer time assessment (Lynch et al, 2014; Wijndaele et al, 2014). Such differences in the assessment of domain-specific sedentary behaviours may have impacted upon the contrasting associations we observed for television watching and time spent on computers with colorectal cancer risk.

The biological mechanisms through which physical activity potentially lowers colon cancer risk are uncertain. Being physically active is associated with less weight gain and body fatness (Lee et al, 2010; May et al, 2012), and lower adiposity is associated with a reduced risk of colorectal cancer (Larsson and Wolk, 2007; Moghaddam et al, 2007). Since body size/adiposity is possibly on the causal pathway linking physical activity and sedentary activities with colorectal cancer, we ran all multivariable models with and without additional adjustment for waist circumference. Adjusting for waist circumference resulted in a modest attenuation of the risk estimates (1-8\%). These results support the hypothesis that the inverse physical activity and colorectal cancer relationship is at least partially mediated through changes in body size and 
composition. As well as influencing weight gain and body fatness, greater physical activity and lower sedentary behaviours have also been associated with lower insulin levels and inflammation (Woods et al, 2006; Dunstan et al, 2007; Helmerhorst et al, 2009), both of which have been linked to the development of colorectal cancer (Kaaks et al, 2000; Terzic et al, 2010; Murphy et al, 2016). Physical activity may also reduce colorectal cancer risk by stimulating digestion and reducing transit time through the intestine (Slattery, 2004; Friedenreich et al, 2010).

The current analysis represents one of the largest single studies investigating the relationships between physical activity levels and sedentary behaviour with colorectal cancer risk to date. The large number of incident colorectal cancer cases allowed analyses by sex and across colorectal subsites, and the detailed phenotypic information collected from UK Biobank participants enabled us to carefully adjust for known colorectal cancer risk factors. A limitation of our analysis is that due to the relatively short followup time (median of 5.6 years) preclinical disease may be influencing the observed associations. However, we observed similar relationships when we excluded cases which occurred during the first 2 years of follow-up. In addition, a recent large participant level pooled analysis reported similar magnitude inverse relationships between leisure-time physical activity and colorectal cancer when stratified by follow-up period $(<5$ years and $\geqslant 5$ years) (Moore et al, 2016), which suggests our results observed in a relatively short follow-up time may not be substantially influenced by preclinical disease. Another limitation of the study is that physical activity and sedentary behaviour information was self-reported by participants at the baseline questionnaire. Recently, the UK Biobank has objectively measured activity levels using 7-day accelerometers, in over 100000 participants (Doherty et al, 2017). Currently, the follow-up time since these accelerometer measurements were collected is too short to study incident colorectal cancer, but these data will offer unique future insights into the physical activity and cancer relationship.

In conclusion, in this prospective analysis of UK Biobank participants, total physical activity levels were inversely associated with colon cancer risk, with no heterogeneity found between tumours located in the proximal colon and distal colon. Sedentary behaviour, as indicated by television watching time, was associated with an elevated colon cancer risk. These findings add to the large body of evidence which supports the promotion of physical activity in population-wide cancer prevention programmes.

\section{ACKNOWLEDGEMENTS}

This work has been conducted using the UK Biobank Resource under Application Number 25897 and we express our gratitude to the participants and those involved in building the resource. UK Biobank is an open access resource. Bona fide researchers can apply to use the UK Biobank data set by registering and applying at http://www.ukbiobank.ac.uk/register-apply/. KEB was supported by a Girdlers' New Zealand Health Research Council Fellowship.

\section{CONFLICT OF INTEREST}

The authors declare no conflict of interest.

\section{REFERENCES}

Ainsworth BE, Haskell WL, Herrmann SD, Meckes N, Bassett DRJ, TudorLocke C, Greer JL, Vezina J, Whitt-Glover MC, Leon AS (2011) Compendium of physical activities: a second update of codes and MET values. Med Sci Sports Exerc 43: 1575-1581.
Allen N, Sudlow C, Downey P, Peakman T, Danesh J, Elliott P, Gallacher J, Green J, Matthews P, Pell J, Sprosen T, Collins R (2012) UK Biobank: current status and what it means for epidemiology. Health Policy Technol 1: 123-126.

Burón Pust A, Alison R, Blanks R, Pirie K, Gaitskell K, Barnes I, Gathani T, Reeves G, Beral V, Green J. Million women study, C (2017) Heterogeneity of colorectal cancer risk by tumour characteristics: large prospective study of UK women. Int J Cancer 140: 1082-1090.

Craig CL, Marshall AL, Sjöström M, Bauman AE, Booth ML, Ainsworth BE, Pratt M, Ekelund U, Yngve A, Sallis JF, Oja P (2003) International physical activity questionnaire: 12-Country reliability and validity. Med Sci Sports Exerc 35: 1381-1395.

Doherty A, Jackson D, Hammerla N, Plötz T, Olivier P, Granat MH, White T, VAN Hees VT, Trenell MI, Owen CG, Preece SJ, Gillions R, Sheard S, Peakman T, Brage S, Wareham NJ (2017) Large scale population assessment of physical activity using wrist worn accelerometers: the UK biobank study. PLOS ONE 12: e0169649.

Dunstan DW, Salmon J, Healy GN, Shaw JE, Jolley D, Zimmet PZ, Owen N (2007) Association of television viewing with fasting and 2-h postchallenge plasma glucose levels in adults without diagnosed diabetes. Diabetes Care 30: $516-522$.

Friedenreich C, Norat T, Steindorf K, Boutron-Ruault MC, Pischon T, Mazuir M, Clavel-Chapelon FO, Linseisen J, Boeing H, Bergman M, Johnsen NF, Tjonneland A, Overvad K, Mendez M, Quiros JR, Martinez C, Dorronsoro M, Navarro C, Gurrea AB, Bingham S, Khaw KT, Allen N, Key T, Trichopoulou A, Trichopoulos D, Orfanou N, Krogh V, Palli D, Tumino R, Panico S, Vineis P, Bueno-De-Mesquita HB, Peeters PHM, Monninkhof E, Berglund G, Manjer J, Ferrari P, Slimani N, Kaaks R, Riboli E (2006) Physical activity and risk of colon and rectal cancers: the European prospective investigation into cancer and nutrition. Cancer Epidemiol Biomark Prev 15: 2398-2407.

Friedenreich CM, Neilson HK, Lynch BM (2010) State of the epidemiological evidence on physical activity and cancer prevention. Eur J Cancer 46: 2593-2604.

Helmerhorst HJF, Wijndaele K, Brage S, Wareham NJ, Ekelund U (2009) Objectively measured sedentary time may predict insulin resistance independent of moderate- and vigorous-intensity physical activity. Diabetes 58: 1776-1779.

Howard RA, Freedman DM, Park Y, Hollenbeck A, Schatzkin A, Leitzmann MF (2008) Physical activity, sedentary behavior, and the risk of colon and rectal cancer in the NIH-AARP Diet and Health Study. Cancer Causes Control 19: 939-953.

Howley ET (2001) Type of activity: resistance, aerobic and leisure versus occupational physical activity. Med Sci Sports Exerc 33: S364-S369.

IARC-WHO (2015) GLOBOCAN 2012: Estimated Cancer Incidence, Mortality and Prevalence Worldwide in 2012.

IPAQ research committee (2005) Guidelines for Data Processing and Analysis of the International Physical Activity Questionnaire (IPAQ) - Short and Long Forms.

Kaaks R, Toniolo P, Akhmedkhanov A, Lukanova A, Biessy C, Dechaud H, Rinaldi S, Zeleniuch-Jacquotte A, Shore RE, Riboli E (2000) Serum C-peptide, insulin-like growth factor (IGF)-I, IGF-binding proteins, and colorectal cancer risk in women. J Natl Cancer Inst 92: 15921600.

Larsson SC, Wolk A (2007) Obesity and colon and rectal cancer risk: a metaanalysis of prospective studies. Am J Clin Nutr 86: 556-565.

Lee I, Djoussé L, Sesso HD, Wang L, Buring JE (2010) Physical activity and weight gain prevention. J Am Med Assoc 303: 1173-1179.

Lee K-J, Inoue M, Otani T, Iwasaki M, Sasazuki S, Tsugane S (2007) Physical activity and risk of colorectal cancer in Japanese men and women: the Japan Public Health Center-based prospective Study. Cancer Causes Control 18: 199-209.

Limsui D, Vierkant RA, Tillmans LS, Wang AH, Weisenberger DJ, Laird PW, Lynch CF, Anderson KE, French AJ, Haile RW, Harnack LJ, Potter JD, Slager SL, Smyrk TC, Thibodeau SN, Cerhan JR, Limburg PJ (2010) Cigarette smoking and colorectal cancer risk by molecularly defined subtypes. J Natl Cancer Inst 102: 1012-1022.

Lynch BM, Friedenreich CM, Khandwala F, Liu A, Nicholas J, Csizmadi I (2014) Development and testing of a past year measure of sedentary behavior: the SIT-Q. BMC Public Health 14: 899.

May AM, Romaguera D, Travier N, Ekelund U, Bergmann MM, Kaaks R, Teucher B, Steffen A, Boeing H, Halkjaer J, Tjonneland A, Jakobsen MU, Overvad K, Dartois L, Fagherazzi G, Boutron-Ruault M-C, Quirós JR, Agudo 
A, Gonzalez C, Sánchez M-J, Amiano P, Huerta J-M, Ardanaz E, Wareham NJ, Crowe FL, Naska A, Orfanos P, Trichopoulou A, Palli D, Agnoli C, Tumino R, Vineis P, Panico S, Bueno-De-Mesquita HB, Verschuren M, Drake I, Sonestedt E, Braaten T, Rinaldi S, Romieu I, Slimani N, Norat T, Riboli E, Peeters PHM (2012) Combined impact of lifestyle factors on prospective change in body weight and waist circumference in participants of the EPIC-PANACEA study. PLOS ONE 7: e50712.

Moghaddam AA, Woodward M, Huxley R (2007) Obesity and risk of colorectal cancer: a meta-analysis of 31 studies with 70,000 events. Cancer Epidemiol Biomark Prev 16: 2533-2547.

Moore SC, Lee IM, Weiderpass E, Campbell PT, Sampson JN, Kitahara CM, Keadle SK, Arem H, Berrington De Gonzalez A, Hartge P, Adami HO, Blair CK, Borch KB, Boyd E, Check DP, Fournier A, Freedman ND, Gunter M, Johannson M, Khaw KT, Linet MS, Orsini N, Park Y, Riboli E, Robien K, Schairer C, Sesso H, Spriggs M, VAN Dusen R, Wolk A, Matthews CE, Patel AV (2016) Association of leisure-time physical activity with risk of 26 types of cancer in 1.44 million adults. JAMA Int Med 176: 816-825.

Murphy N, Cross AJ, Abubakar M, Jenab M, Aleksandrova K, Boutron-Ruault MC, Dossus L, Racine A, Kuhn T, Katzke VA, Tjonneland A, Petersen KEN, Overvad K, Quiros JR, Jakszyn P, Molina-Montes E, Dorronsoro M, Huerta JM, Barricarte A, Khaw KT, Wareham N, Travis RC, Trichopoulou A, Lagiou P, Trichopoulos D, Masala G, Krogh V, Tumino R, Vineis P, Panico S, Bueno-De-Mesquita HB, Siersema PD, Peeters PH, Ohlsson B, Ericson U, Palmqvist R, Nystrom H, Weiderpass E, Skeie G, Freisling H, Kong SY, Tsilidis K, Muller DC, Riboli E, Gunter MJ (2016) A nested case - control study of metabolically defined body size phenotypes and risk of colorectal cancer in the european prospective investigation into cancer and nutrition (EPIC). PLoS Med 13: e1001988.

Office for National Statistics (2006) The Time Use Survey, 2005. How We Spend Our Time London.

Owen N, Leslie E, Salmon J, Fotheringham MJ (2000) Environmental determinants of physical activity and sedentary behavior. Exerc Sport Sci Rev 28: 153-158.

Schmid D, Leitzmann MF (2014) Television viewing and time spent sedentary in relation to cancer risk: a meta-analysis. J Natl Cancer Inst 106: dju098dju098.
Schoenfeld D (1982) Partial residuals for the proportional hazards regression model. Biometrika 69: 239-241.

Slattery ML (2004) Physical activity and colorectal cancer. Sports Med 34: 239-252.

Terzic J, Grivennikov S, Karin E, Karin M (2010) Inflammation and colon cancer. Gastroenterology 138: 2101-2114.e5.

UK-Biobank (2010) UK Biobank. Protocol for a large-scale prospective epidemiological resources [Online] http://www.ukbiobank.ac.uk/wpcontent/uploads/2011/11/UK-Biobank-Protocol.pdfAccessed 10 July 2016.

WCRF-AICR (2011) World Cancer Research Fund-American Institute for Cancer Research. Continuous Update Project Report Summary. Food, Nutrition, Physical Activity, and the Prevention of Colorectal Cancer.

Wei EK, Colditz GA, Giovannucci EL, WU K, Glynn RJ, Fuchs CS, Stampfer M, Willett W, Ogino S, Rosner B (2017) A comprehensive model of colorectal cancer by risk factor status and subsite using data From the nurses' health study. Am J Epidemiol 185: 224-237.

Wei EK, Giovannucci E, WU K, Rosner B, Fuchs CS, Willett WC, Colditz GA (2004) Comparison of risk factors for colon and rectal cancer. Int J Cancer 108: 433-442.

Wiecha JL, Peterson KE, Ludwig DS, Kim J, Sobol A, Gortmaker SL (2006) When children eat what they watch: impact of television viewing on dietary intake in youth. Arch Pediatr Adolesc Med 160: 436-442.

Wijndaele K, DE Bourdeaudhuij I, Godino JG, Lynch BM, Griffin SJ, Westgate K, Brage S (2014) Reliability and validity of a domain-specific last 7-d sedentary time questionnaire. Med Science in Sports Exerc 46: 1248-1260.

Wolin KY, Yan Y, Colditz GA, Lee IM (2009) Physical activity and colon cancer prevention: a meta-analysis. Br J Cancer 100: 611-616.

Woods JA, Vieira VJ, Keylock KT (2006) Exercise, inflammation, and innate immunity. Neurol Clin 24: 585-599.

This work is published under the standard license to publish agreement. After 12 months the work will become freely available and the license terms will switch to a Creative Commons AttributionNonCommercial-Share Alike 4.0 Unported License.

Supplementary Information accompanies this paper on British Journal of Cancer website (http://www.nature.com/bjc) 